\title{
Stability Analysis of Plane Vibrations of a Satellite in a Circular Orbit
}

\author{
H. Yehia*, H. Nabih \\ Department Of Mathematics, Faculty of Science, Mansoura University, Mansoura 35516, Egypt
}

Received: 26 June 2013 / Accepted: 05 August 2013

*Corresponding author (Tel: +20 502242388 Fax: +20502246781, email: hyehia@ mans.edu.eg)

\begin{abstract}
In this paper, we analyze for stability the problem of planar vibrational motion of a satellite about its centre of mass. The satellite is dynamically symmetric whose center of mass is moving in a circular orbit. The in-plane motion is a simple pendulum-like motion in which the axis of symmetry of the satellite remains in the orbital plane. It is expressed in terms of elliptic functions of time. Using Routh's equations we study the orbital stability of planar vibrations of the satellite, in the sense that the stable in-plane motions remain under perturbation very near to the orbital plane. The linearized equation for the out-of-plane motion takes the form of a Hill's equation. Detailed analysis of stability using Floquet theory is performed analytically and numerically. Zones of stability and instability are illustrated graphically in the plane of the two parameters of the problem: the ratio of moments of inertia and the amplitude of the unperturbed motion.
\end{abstract}

Keywords: Satellite, Plane vibrations, Stability, Floquet theory, Periodic solutions

\section{Introduction}

The field of dynamics of a rigid body is one of the most complicated fields of theoretical mechanics. Even in its simplest case, that of motion of a body about a fixed point in a uniform gravity field, it belongs to the type of nonintegrable dynamics (see e.g. [1], [2]). Nevertheless, it is an inevitable component of the study of many important systems in science and technology. An example is the problem of study of the translational-rotational motion of a satellite. If the last problem is considered in its full generality, the equations of translational motion of the satellite and its rotational motion are coupled together. It was shown that for an artificial satellite a possible solution represents motion in a circular orbit (e.g. [3]). In that case the rotational motion of the satellite about its centre of mass relative to the orbital coordinate system can be described as motion about a fixed point of a rigid body acted upon by nonsymmetric potential and gyroscopic forces [4].

Suppose that the satellite is a dynamically symmetric rigid body and its center of mass moves in a circular orbit. A possible solution of the equations of motion represents what is called in-plane motion. That is a pendulum-like motion in which the axis of symmetry of the satellite remains all the time in the orbital plane (see e.g. [3]). The aim of this paper is to study the stability of the planar periodic motion, in particular, the 
stability of vibration motion. Markeev [5] was the first to study the problem in the above formulation. He studied the orbital stability of the planar periodic motion for dynamically symmetric satellite whose polar axis of the ellipsoid of inertia is shorter than the equatorial ones. But, diagrams of stability contained some imperfections because of low accuracy of numerical calculations. Akulenko, Nesterov and Shmatkov [6] pointed out these imperfections. Markeev and Bardin [7] studied the problem of stability of the planer periodic motions in the nonlinear setting using the normal forms of Birkhoff. Such analysis can be performed only numerically.

In this paper, we study the stability of plane vibrational motions of a satellite in a circular orbit. The problem is reduced to a form of Hill's equation, i.e. a single second-order linear differential equation with a periodic coefficient. It turned out that this equation is a generalization of Lame's equation of the first rank. To deal with this equation we combine Floquet theory [8] with the method devised by Ince for Lame's equation, which consists in finding primitive periodic solutions in the form of Fourier series expansions. Point sets in the space of parameters, corresponding to those solutions separate zones of stability and instability. We obtain a detailed picture of those zones, analytically and in a purely numerical treatment. Both analyses are in complete agreement.

\section{Mathematical Formulation}

\section{Description of motion}

Denote by $O$ the center of the Earth and by $O^{\prime}$ the current position of the center of mass of the satellite describing a circular orbit of radius $\mathrm{R}$ with centre at $O$ see Fig.1. Let $O X Y Z$ be an inertial frame of reference, $O^{\prime} x_{1} y_{1} z_{1}$ be the orbital system with $x_{1}$ - axis along $0 O^{\prime}$, $y_{1}$-along the tangent to the orbit in the direction of motion of the satellite and $z_{1}-$ in the direction orthogonal to the orbital plane and let $O^{\prime} x y z$ be the system of central principal axes of the satellite with moments of inertia A,B and $\mathrm{C}$, respectively. Let also $\boldsymbol{\alpha}_{s} \boldsymbol{\beta}_{s} \gamma$ be the three unit vectors in the directions of $x_{1}, y_{1}, z_{1}$ and $\omega$ the angular velocity vector of the satellite relative to the orbital system, all components being referred to the body system $O^{\prime} x y z$. To describe the orientation of the satellite relative to the orbital frame we shall use Euler's angle $\psi$ the angle of precession around the $z_{1}-$ axis, $\theta$ the angle between the axis of the body and the orbital plane (so that the nutation angle between $z$ and $z_{1}-$ axis is $\frac{\pi}{2}-\theta$ ) and $\varphi$ the angle of proper rotation of the satellite around its $z$ - axis.

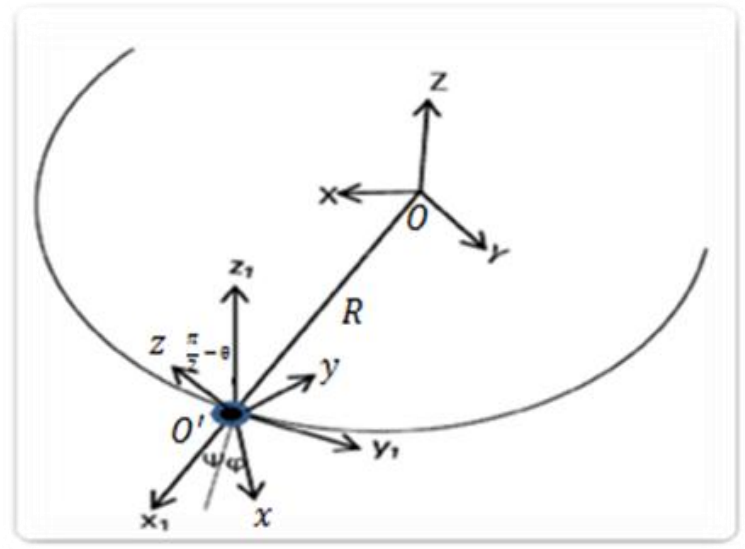

Fig. 1 Euler's angles for describing the orientation of the satellite

In those variables we can write $\alpha=(\cos \psi \cos \phi-\sin \theta \sin \phi \sin \psi$ $-\cos \psi \sin \phi-\sin \theta \cos \phi \sin \psi$, $\cos \theta \sin \psi)$

$\beta=\left(\sin \psi \cos \phi+\sin \theta \sin \phi \cos \psi_{p}\right.$ $-\sin \psi \cos \phi+\sin \theta \sin \phi \cos \psi_{n}$ $-\cos \theta \cos \psi)$

$\gamma=\left(\cos \theta \sin \phi_{x} \cos \theta \cos \phi_{x} \sin \theta\right)$

$\omega=(\dot{\psi} \cos \theta \sin \phi-\cos \phi, \dot{\psi} \cos \theta \cos \phi$

$$
+\sin \phi, \dot{\psi} \sin \phi+\dot{\phi})
$$

In what follows we study the rotational motion of the satellite about its centre of mass. It is supposed that the rotational motion is independent of the orbital motion of the satellite with uniform angular speed $\Omega$ (say), given by the 
expression $\Omega^{2}=\frac{\mu}{R^{3}}$ where $\mu$ is Gauss' constant of the Earth [3]. The Lagrangian of the rotational motion can be written as

$L=\frac{1}{2} w I \cdot w-V$

$w=\omega+\Omega Y$

where $\boldsymbol{w}$ the absolute angular velocity of the satellite and $\mathrm{V}$ its potential function in the gravitational field of Earth. A normally acceptable approximate form (see e.g. Beletskii [3]) is:

$V=\frac{3 \mu}{2 R^{3}} \boldsymbol{\alpha} I \cdot \boldsymbol{\alpha}=\frac{3}{2} \Omega^{2} \boldsymbol{\alpha} \cdot \boldsymbol{\alpha}$

Substituting (2) and (3) in (1) we get

$L=\frac{1}{2}\left(A p^{2}+B q^{2}+C r^{2}\right)$

$+\Omega\left(A p \gamma^{1}+B q \gamma^{2}+C r \gamma^{3}\right)$

$+\frac{a^{2}}{2}(\gamma I \cdot \gamma+3 \alpha I \cdot \alpha)$

The equation of motion of the satellite relative to the orbital frame, corresponding to the Lagrangian can be written in the Euler$\dot{G}+\omega \times(G-2 \Omega Y \bar{I})=$

$$
3 \Omega^{2} \alpha \times \alpha I-\Omega^{2} \gamma \times \gamma I
$$

$\ddot{\alpha}+\omega \times \alpha=0$

$$
\beta+\omega \times \beta=0
$$

$\dot{\gamma}+\omega \times \gamma=0$

where $\bar{I}=\frac{1}{2}(\operatorname{Tr} I) \delta-I$. This system admits Jacobi's integral

$\frac{1}{2} \omega I \cdot \omega+\frac{\Omega^{2}}{2}(3 \alpha I \cdot \alpha-\gamma I \cdot \gamma)=h$

It was noted in [4] that this system of equations (6) of motion of the satellite on a circular orbit is form-equivalent to the equations of motion of an electrically charged rigid body about a fixed point of it, while acted upon by gravitational, electric and magnetic fields. The system (5) admits a simple solution, which represents motion of the satellite with two of its principal axes lying always in the orbital plane and the third orthogonal to it. Let the last axis be the $\boldsymbol{x}$-axis. It is not hard to see that this solution is

$\omega=\omega \gamma_{g} \omega=\hat{\psi}$,

$\boldsymbol{\alpha}=\left(0, \cos \psi_{y} \sin \psi\right)$,

$\left.-\sin \psi_{3} \cos \psi\right){ }_{\jmath} \gamma=(1,0,0)$

When $\dot{\psi}=0$ the satellite takes the relative equilibrium position with one of its axes directed to the Earth's centre and another directed along the tangent to the orbit. In general we have two types of motion: vibration and rotation. Each is centered about one of the equilibrium positions. This occurs according to the ratio $\alpha=\frac{C}{A}$ of moment of inertia of the satellite, restricted by the triangle inequality to the interval $[0,2]$. The subinterval

i) $0<a<1$ corresponds to the case $c<A=B$

ii) $1<\alpha<2$ to the case $C>A=B$.

For the first case the satellite is moving around the tangent of the orbital plane and the second cases the satellite is moving around $O O^{\prime}$.

\section{Equation of motion}

We may express the Lagrangian of the problem in the form:

$$
\begin{aligned}
L= & \frac{1}{2} A\left(p^{2}+q^{2}\right)+\frac{1}{2} C r^{2} \\
& +\Omega\left[A\left(p \gamma_{1}+q \gamma_{2}\right)+C r \gamma_{3}\right] \\
& +\frac{\Omega^{2}}{2}\left[A\left(\gamma_{1}^{2}+\gamma_{2}^{2}\right)\right. \\
& \left.+C \gamma_{3}^{2}+(C-A)\left(1-3 \alpha_{3}^{2}\right)\right]
\end{aligned}
$$

Case $i(0<\alpha<1)$

Under the condition $i$ the Lagrangian is $\left(\Psi=\frac{\pi}{2}+\psi\right)$ 


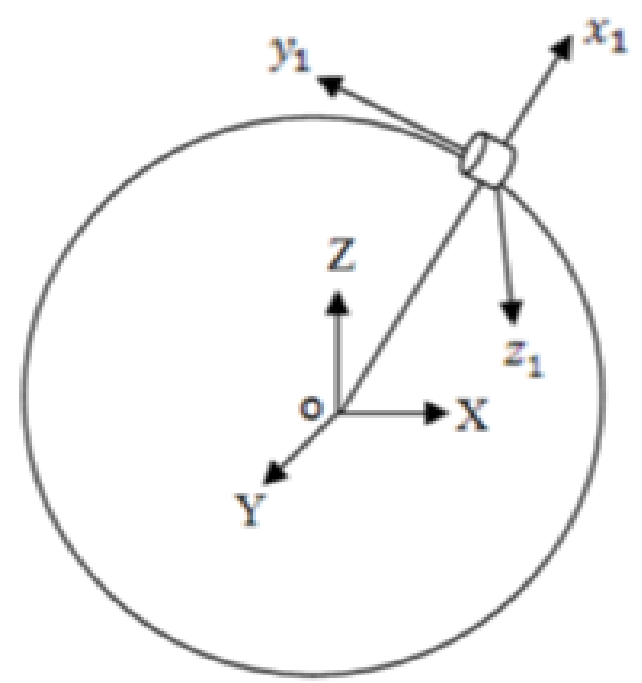

Fig. 2 Direction of the satellite axes for the case I $(0<\alpha<1)$

$L=\left[\frac{A}{2} \cos ^{2} \theta+\frac{C}{2} \sin ^{2} \theta\right] \Psi^{2}$

$+\left[\Omega\left(A \cos ^{2} \theta+C \sin ^{2} \theta\right)+C \varphi \sin \theta\right] \Psi+\frac{A}{2} \dot{\theta}^{2}$ $+\Omega \dot{\varphi} C \sin \theta+\frac{C}{2} \dot{\varphi}^{2}$

$+\frac{\Omega^{2}}{2}(A-C)\left[\cos ^{2} \theta+3 \cos ^{2} \Psi \cos ^{2} \theta\right]$

Now, we note that $\varphi$ is a cyclic coordinate where:

$$
\begin{aligned}
& p_{\varphi}=\frac{\partial L}{\partial \dot{\varphi}}=C\left[\left(\dot{\Psi}^{\prime}+\Omega\right) \sin \theta+\dot{\varphi}\right]=C_{1} \\
& \dot{\varphi}=C_{1}-(\dot{\Psi}+\Omega) \sin \theta
\end{aligned}
$$

Next, we ignore $\varphi$ and construct the Routhian: $R=\frac{A}{2}\left[\dot{\theta}^{2}+\mathscr{\Psi}^{2} \cos ^{2} \theta\right]$

$$
\begin{aligned}
& +\left[\Omega A \cos ^{2} \theta+C C_{1} \sin \theta\right]{ }^{3} \\
& +\frac{\Omega^{2}}{2}[C-A+ \\
& \left.A \cos ^{2} \theta+3(A-C) \cos ^{2} \Psi \cos ^{2} \theta\right] \\
& +\frac{C C_{1}}{2}\left(2 \Omega \sin \theta-C_{1}\right)
\end{aligned}
$$

The satellite can perform the in-plane motions only if the real constant $C_{1}=0$.

According to this condition the Routhian takes the form:

$$
\begin{aligned}
R= & \frac{A}{2}\left[\dot{\theta}^{2}+\Psi^{2} \cos ^{2} \theta\right] \\
& +\Omega A \cos ^{2} \theta \Psi+\frac{\Omega^{2}}{2}[C-A+ \\
& \left.A \cos ^{2} \theta+3(A-C) \cos ^{2} \Psi \cos ^{2} \theta\right]
\end{aligned}
$$

The equations of motion of the satellite in this case become

$$
\begin{aligned}
& A \ddot{\Psi} \cos ^{2} \theta-2 A \dot{\theta}(\Psi+\Omega) \sin \theta \cos \theta \\
& +3 \Omega^{2}(A-C) \cos ^{2} \theta \sin \Psi \cos \Psi=0 \\
& A \ddot{\theta}+A \Psi^{2} \sin \theta \cos \theta+2 A \Omega \Psi^{2} \sin \theta \cos \theta \\
& +3 \Omega^{2}(A-C) \cos ^{2} \Psi \sin \theta \cos \theta+ \\
& \Omega^{2} A \sin \theta=0
\end{aligned}
$$

Defining the non-dimensional time $\tau=\Omega t$ and denoting derivatives with respect to $\tau$ by 0 the non-dimensional equations of motion become:

$$
\begin{aligned}
& \Psi^{\omega} \cos ^{2} \theta-2 \theta^{\circ}\left(\Psi^{\sigma}+1\right) \sin \theta \cos \theta \\
& +3(1-\alpha) \cos ^{2} \theta \sin \Psi \cos \Psi=0 \\
& \theta^{\omega}+\Psi^{2} \sin \theta \cos \theta+2 \Psi^{\sigma} \sin \theta \cos \theta \\
& +(1-\alpha) \cos ^{2} \Psi \sin \theta \cos \theta \\
& +\sin \theta=0
\end{aligned}
$$

Case ii $(1<\alpha<2)$

In this case the equatorial axis remains approximately orthogonal to the plane of the orbit and the polar axis performs small angular oscillations about this plane so we shall use Euler's angle $\psi$ the angle of precession around the $z_{1}$-axis (see Fig. 3).

Now, the Lagrangian function takes the form:

$$
\begin{aligned}
L= & \left(\frac{A}{2} \cos ^{2} \theta+\frac{C}{2} \sin ^{2} \theta\right) \psi^{2}+ \\
& {\left[\Omega\left(A \cos ^{2} \theta+C \sin ^{2} \theta\right)+C \dot{s i n} \theta\right] \psi+} \\
& \frac{A}{2} \dot{\theta}^{2}+\Omega \dot{\varphi} C \sin \theta+\frac{C}{2} \dot{\varphi}^{2}- \\
& \frac{\Omega^{2}}{2}\left[\begin{array}{c}
4(C-A) \cos ^{2} \theta-2 C+ \\
A-3(C-A) \cos ^{2} \psi \cos ^{2} \theta
\end{array}\right]
\end{aligned}
$$




$$
p_{\varphi}=\frac{\partial L}{\partial \dot{\varphi}}=C[(\dot{\psi}+\Omega) \sin \theta+\dot{\varphi}]=C_{2}
$$

and we get the Routhian

$$
R=\frac{A}{2}\left[\hat{\theta}^{2}+\hat{\psi}^{2} \cos ^{2} \theta\right]+\left[\Omega A \cos ^{2} \theta+C C_{2} \sin \theta\right] \psi
$$

$$
\begin{aligned}
& +\frac{\Omega^{2}}{2}\left[C-A+(4 A-3 C) \cos ^{2} \theta+\right. \\
& \left.3(C-A) \cos ^{2} \Psi \cos ^{2} \theta\right] \\
& +\frac{C C_{2}}{2}\left(2 \Omega \sin \theta-C_{2}\right)
\end{aligned}
$$

Then the equations of motion are:

$A \psi \cos ^{2} \theta+\left[C C_{2} \cos \theta-2 A(\psi+\Omega) \sin \theta \cos \theta\right] \hat{\theta}$ $+3 \Omega^{2}(C-A) \cos ^{2} \theta \sin \psi \cos \psi=0$

$A \vec{\theta}+A \dot{\psi}^{2} \sin \theta \cos \theta-\left[C C_{2}-2 A \Omega \sin \theta\right] \dot{\psi} \cos \theta$ $+3 \Omega^{2}(C-A) \cos ^{2} \psi \sin \theta \cos \theta-C C_{2} \Omega \cos \theta$ $+\Omega^{2}(4 A-3 C) \sin \theta \cos \theta=0$

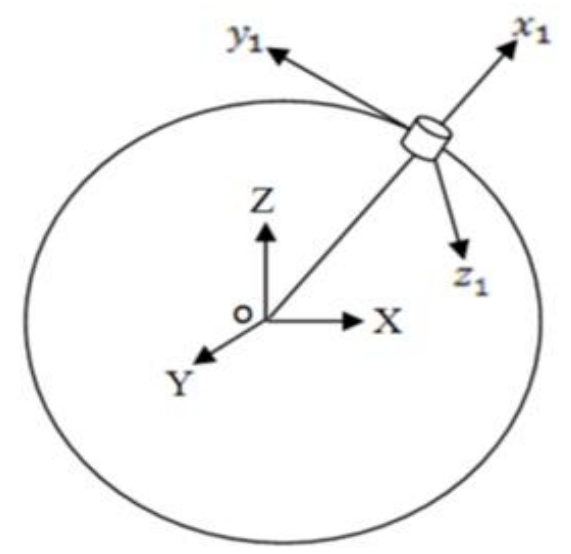

Fig. 3 Direction of the satellite axes for the case II $(1<\alpha<2)$

And in terms of the non-dimensional time $\psi^{\omega} \cos ^{2} \theta-\left[\alpha C_{2} \cos \theta-2\left(\psi^{\circ}+1\right) \sin \theta \cos \theta\right] \theta$ $+3(\alpha-1) \cos ^{2} \theta \sin \psi \cos \psi=0$

$$
\theta^{\varpi}+\psi^{2} \sin \theta \cos \theta-
$$$$
\left(\alpha C_{2}-2 \sin \theta\right) \psi^{\circ} \cos \theta+3(\alpha-1) \cos ^{2} \psi \sin \theta \cos \theta
$$$$
+(4-3 \alpha) \sin \theta \cos \theta-\frac{\alpha C_{2}}{\Omega} \cos \theta=0
$$

The equations (14), (15), (21) and (22) describe the motion of the satellite relative to the orbital frame.

\section{Solution for in-plane motion}

Assume that $0<\alpha<1$, by backing to equation (14) the in-plane motion $\theta=0$ is described by

$$
\Psi^{\omega}+3(1-\alpha) \sin \Psi \cos \Psi=0
$$

Integrating this equation we obtain

$\Psi^{2}+3(1-\alpha) \sin ^{2} \Psi=h$

Where $h$ is a measure of the energy of the inplane motion. We will show that the motion is classified as follow (see Fig. 4):

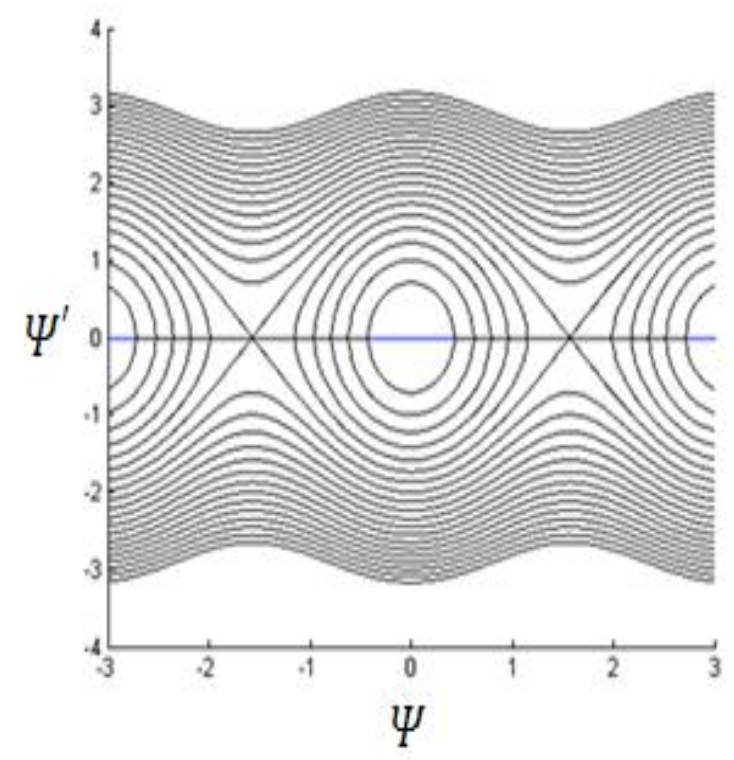

\begin{tabular}{|c|c|}
\hline $\mathrm{h}<0$ & no motion \\
\hline $\mathrm{h}=0$ & equilibrium \\
\hline $\mathrm{h}>\mathrm{h}<3\left(1-\frac{C}{A}\right)$ & periodic oscillation \\
\hline & $\begin{array}{c}\text { continuous rotation and } \\
\text { the direction of the } \\
\text { rotation determined by the } \\
\text { sign of the initial rotation } \\
\text { rate }\end{array}$ \\
\hline $\mathrm{h}=3\left(1-\frac{C}{A}\right)$ & $\begin{array}{c}\text { separate the zones of } \\
\text { oscillations and liberations }\end{array}$ \\
\hline
\end{tabular}

Fig. 4 Phase portrait for in-plane motion assuming zero out-of-plane inclination

From equation (24) under the condition $(0<\alpha<1)$

$$
\Psi^{\sigma}= \pm \sqrt{h-3(1-\alpha) \sin ^{2} \Psi}
$$

and on separation of variables

$$
\begin{aligned}
& \int \frac{d \Psi}{\sqrt{1-k_{1}^{2} \sin ^{2} \Psi}}= \pm \sqrt{h} \int d \tau \\
& k_{1}^{2}=\frac{3}{h}(1-\alpha)
\end{aligned}
$$


If $\| k_{1} \rrbracket>1, h<3(1-\alpha)$

we define $v_{1}=\frac{1}{k_{1}}$

$$
\begin{aligned}
& \Psi(\tau)=\sin ^{-1}\left(v_{1} \operatorname{sn}\left(\sqrt{3(1-\alpha)} \pi, v_{1}\right)\right), \\
& \Psi^{\sigma}(\tau)=\sqrt{h} \operatorname{cn}\left(\sqrt{3(1-\alpha)} \tau, v_{1}\right) \\
& \text { if }\left\|k_{1}\right\|<1, \quad h>3(1-\alpha) \\
& \Psi(\tau)=\operatorname{am}\left( \pm \sqrt{h} \tau, k_{1}\right), \\
& \Psi^{\sigma}(\tau)= \pm \sqrt{h} d n\left(\sqrt{h} \tau_{,} k_{1}\right)
\end{aligned}
$$

For second case when we study the in-plane motion $\theta=0$ and $C_{2}=0$ then

$\psi^{\omega}+3(\alpha-1) \sin \psi \cos \psi=0$

$\psi^{2}+3(\alpha-1) \sin ^{2} \psi=h$

$\psi^{\circ}= \pm \sqrt{h-3(\alpha-1) \sin ^{2} \psi}$

$$
\int \frac{d \psi}{k_{2}^{2}=\frac{3}{h}(\alpha-1)}= \pm \sqrt{h} \int d \tau
$$

$$
\begin{aligned}
& \text { if }\left\|k_{2}\right\|<1, h>3(\alpha-1) \\
& \psi(\tau)=a m\left( \pm \sqrt{h} \tau, k_{2}\right) \text {, } \\
& \psi^{\circ}(\tau)= \pm \sqrt{h} d n\left(\sqrt{h} \pi, k_{2}\right) \\
& \text { if }\left\|k_{2}\right\|>1, h<3(\alpha-1), v_{2}=\frac{1}{k_{2}} \\
& \psi(\tau)=\sin ^{-1}\left(v_{2} \operatorname{sn}\left(\sqrt{3(\alpha-1)} \pi, v_{2}\right)\right), \\
& \psi^{\circ}(\tau)=\sqrt{h} c n\left(\sqrt{3(\alpha-1)} \pi, v_{2}\right)
\end{aligned}
$$

\section{Studying of Vibration motion}

\section{Stability of in-plane motion}

The linear stability equation w. r. to $\theta$ is

$$
\begin{aligned}
& \theta^{\varpi}+\left[\Psi^{2}+1+2 \Psi^{\sigma}+3(1-\alpha)-3(1-\alpha) \sin ^{2} \Psi\right] \theta \\
& =0 \quad(0<\alpha<1) \\
& \theta^{\varpi}+\left[\Psi^{2}+1+2 \psi^{\sigma}-3(\alpha-1) \sin ^{2} \Psi\right] \theta=0 \\
& (1<\alpha<2)
\end{aligned}
$$

Let $w=\sqrt{3(1-\alpha)} \tau$ and $\Psi=a m(w)$. Using (26) and (32) we get

$$
\frac{d^{2} \theta}{d w^{2}}+\left[\begin{array}{c}
1+v_{1}^{2}+\frac{1}{3(1-\alpha)}- \\
2 v_{1}^{2} \operatorname{sn}^{2}\left(w_{3} v_{1}\right)+ \\
\frac{w w_{1}}{\sqrt{g(1-\alpha)}} c n\left(w_{3} v_{1}\right)
\end{array}\right] \theta=0
$$

In trigonometric form with $\sin \Psi=v_{1} \sin \beta$

$$
\begin{aligned}
& \left(1-v_{1}^{2} \sin ^{2} \beta\right) \frac{d^{2} \theta}{d \beta^{2}}- \\
& v_{1}^{2} \sin \beta \cos \beta \frac{d \theta}{d \beta}+ \\
& \left(1+v_{1}^{2}+\frac{1}{3(1-\alpha)}-2 v_{1}^{2} \sin ^{2} \beta\right. \\
& \left.+\frac{2 v_{1}}{\sqrt{\pi(1-\alpha)}} \cos \beta\right) \theta=0
\end{aligned}
$$

This equation can be transformed to hill's equation as follow:

Let $\theta=g f$

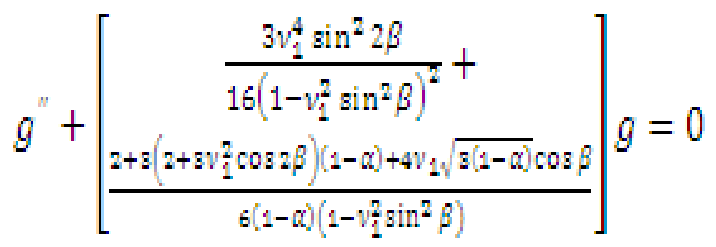

When $\quad w=\sqrt{3(\alpha-1)} \pi$ and $\psi=a m(w)$ then use equations (30) and (33) to get

$$
\frac{d^{2} \theta}{d v^{2}}+\left[\begin{array}{c}
v_{2}^{2}+\frac{1}{3(\alpha-1)}-2 v_{2}^{2} \operatorname{sn}^{2}\left(w_{1} v_{2}\right) \\
+\frac{w v_{2}}{\sqrt{\mathrm{s}(\alpha-1)}} c n\left(w_{3} v_{2}\right)
\end{array}\right] \theta=0
$$

We will use the same previous method to get the trigonometric form

$$
\begin{aligned}
& \sin \psi=v_{2} \sin \beta \quad \& \quad \psi^{\sigma}=\sqrt{h} \cos \beta \\
& \left(1-v_{2}^{2} \sin ^{2} \beta\right) \frac{d^{2} \theta}{d \beta^{2}}-v_{2}^{2} \sin \beta \cos \beta \frac{d \theta}{d \beta} \\
& +\left(v_{2}^{2}+\frac{1}{3(\alpha-1)}-2 v_{2}^{2} \sin ^{2} \beta\right. \\
& +\frac{2 v_{x}}{\sqrt{\mathrm{g}(\alpha-1)} \cos \beta) \theta=0}
\end{aligned}
$$

Transform it to Hill's equation

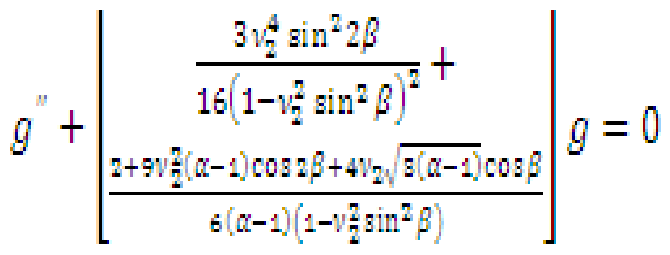


The equations $(36 \& 39)$ are periodic with periods $2 \pi$ and $4 \pi$ and depend on two parameters $\left(\alpha, \psi_{0}\right)$. In the following subsection we deduce the equations of the boundaries between zones of stability and instability in the plane of those parameters.

The stability of vibration motion

Using Floquet's theorem we will study the stability of equation (35) both analytically and numerically. For simplicity we write the equation in the form

$$
\begin{aligned}
& \left(1-v_{1}^{2} \sin ^{2} \beta\right) \frac{d^{2} \theta}{d \beta^{2}}- \\
& v_{1}^{2} \sin \beta \cos \beta \frac{d \theta}{d \beta}+ \\
& \left(b-2 v_{1}^{2} \sin ^{2} \beta+d \cos \beta\right) \theta=0 \\
& b=1+v_{1}^{2}+\frac{1}{3(1-\alpha)}, \quad d=\frac{2 v_{1}}{\sqrt{3(1-\alpha)}}
\end{aligned}
$$

and equation (38) in the form

$$
\left(1-v_{2}^{2} \sin ^{2} \beta\right) \frac{d^{2} \theta}{d \beta^{2}}-
$$$$
v_{2}^{2} \sin \beta \cos \beta \frac{d \theta}{d \beta}+
$$$$
\left(b_{1}-2 v_{2}^{2} \sin ^{2} \beta+d_{1} \cos \beta\right) \theta=0
$$

$b_{1}=v_{2}^{2}+\frac{1}{3(\alpha-1)}, \quad d_{1}=\frac{2 v_{z}}{\sqrt{3(\alpha-1)}}$

The primitive periodic solutions of equations (40) and (41) can be expressed as a Fourier series in one of the forms:

\section{Odd $2 \pi$ periodic solution}

$\theta=\sum_{n=0}^{\infty} A_{n} \sin n \beta$

Substitution this solution in (40), yields the following recurrence relations for coefficients $A_{m}$ $\left(b-1-v_{1}^{2}\right) A_{1}+\frac{d}{2} A_{2}-v_{1}^{2} A_{3}=0$ $\frac{d}{2} A_{1}+\left(b-4+v_{1}^{2}\right) A_{2}+\frac{d}{2} A_{3}-\frac{5}{2} v_{1}^{2} A_{4}=0$ $\frac{d}{2} A_{2}+\left(b-9+\frac{7}{2} v_{1}^{2}\right) A_{3}+\frac{d}{2} A_{4}-\frac{9}{2} v_{1}^{2} A_{5}=0$

For $n \geq 2$

$$
\begin{aligned}
& -\frac{n(2 n-3)}{2} v_{1}^{2} A_{2 n-2}+\frac{d}{2} A_{2 n-1}+ \\
& \left(b-4 n^{2}+\left(2 n^{2}-1\right) v_{1}^{2}\right) A_{2 n}+\frac{d}{2} A_{2 n+1} \\
& -\frac{n(2 n+3)}{2} v_{1}^{2} A_{2 n+2}=0 \\
& -\frac{n(2 n-1)-1}{2} v_{1}^{2} A_{2 n-1}+\frac{d}{2} A_{2 n} \\
& +\left(b-(2 n+1)^{2}+\frac{\left.(2 n+1)^{2}-2\right)}{2} v_{1}^{2}\right) A_{2 n+1} \\
& +\frac{d}{2} A_{2 n+2}-\frac{n(2 n+5)+2}{2} v_{1}^{2} A_{2 n+3}=0
\end{aligned}
$$

For the second case, using (41) gives for coefficients $A_{n}$ the recurrence relations

$$
\begin{aligned}
& \left(b_{1}-1-v_{2}^{2}\right) A_{1}+\frac{d_{1}}{2} A_{2}-v_{2}^{2} A_{3}=0 \\
& \frac{d_{1}}{2} A_{1}+\left(b_{1}-4+v_{2}^{2}\right) A_{2}+\frac{d_{1}}{2} A_{3}-\frac{5}{2} v_{2}^{2} A_{4}=0 \\
& \frac{d_{1}}{2} A_{2}+\left(b_{1}-9+\frac{7}{2} v_{2}^{2}\right) A_{3}+\frac{d_{1}}{2} A_{4}-\frac{9}{2} v_{2}^{2} A_{5}=0
\end{aligned}
$$

For $n \geq 2$

$$
\begin{aligned}
& -\frac{n(2 n-3)}{2} v_{2}^{2} A_{2 n-2}+\frac{d_{1}}{2} A_{2 n-1} \\
& +\left(b_{1}-4 n^{2}+\left(2 n^{2}-1\right) v_{2}^{2}\right) A_{2 n}+\frac{d_{1}}{2} A_{2 n+1} \\
& -\frac{n(2 n+3)}{2} v_{2}^{2} A_{2 n+2}=0 \\
& -\frac{n(2 n-1)-1}{2}\left(2 n^{2}-1\right) v_{2}^{2} A_{2 n-1}+\frac{d_{1}}{2} A_{2 n} \\
& +\left(b_{1}-(2 n+1)^{2}+\frac{\left.(2 n+1)^{2}-2\right)}{2} v_{2}^{2}\right) A_{2 n+1} \\
& +\frac{d_{1}}{2} A_{2 n+2}-\frac{n(2 n+5)+2}{2} v_{2}^{2} A_{2 n+3}=0
\end{aligned}
$$

Even $2 \pi$-periodic solution

Substituting

$\theta=\sum_{m=0}^{\infty} B_{n} \cos n \beta$

in equation (40) yields the recurrence relations for coefficients $B_{n}$ :

$$
\begin{aligned}
& \left(b-v_{1}^{2}\right) B_{0}+\frac{d}{2} B_{1}=0 \\
& d B_{0}+(b-1) B_{1}+\frac{d}{2} B_{2}-v_{1}^{2} B_{3}=0 \\
& v_{1}^{2} B_{0}+\frac{d}{2} B_{1}+\left(b-4+v_{1}^{2}\right) B_{2}+\frac{d}{2} B_{3}-\frac{5}{2} v_{1}^{2} B_{4}=0 \\
& \frac{d}{2} B_{2}+\left(b-9+\frac{7}{2} v_{1}^{2}\right) B_{3}+\frac{d}{2} B_{4}-\frac{9}{2} v_{1}^{2} B_{5}=0
\end{aligned}
$$


For $n \geq 2$

$$
\begin{aligned}
& -\frac{n(2 n-3)}{2} v_{1}^{2} B_{2 n-2}+\frac{d}{2} B_{2 n-1}+ \\
& \left(b-4 n^{2}+\left(2 n^{2}-1\right) v_{1}^{2}\right) B_{2 n}+\frac{d}{2} B_{2 n+1} \\
& -\frac{n(2 n+3)}{2} v_{1}^{2} B_{2 n+2}=0 \\
& -\frac{n(2 n-1)-1}{2} v_{1}^{2} B_{2 n-1}+\frac{d}{2} B_{2 n}+ \\
& \left(b-(2 n+1)^{2}+\frac{\left.(2 n+1)^{2}-2\right)}{2} v_{1}^{2}\right) B_{2 n+1} \\
& +\frac{d}{2} B_{2 n+2}-\frac{n(2 n+5)+2}{2} v_{1}^{2} B_{2 n+3}=0
\end{aligned}
$$

And in (41) leads to the relations

$$
\begin{aligned}
& \left(b_{1}-v_{2}^{2}\right) B_{0}+\frac{d_{1}}{2} B_{1}=0 \\
& d_{1} B_{0}+\left(b_{1}-1\right) B_{1}+\frac{d_{1}}{2} B_{2}-v_{2}^{2} B_{3}=0 \\
& v_{2}^{2} B_{0}+\frac{d_{1}}{2} B_{1}+\left(b_{1}-4+v_{2}^{2}\right) B_{2}+\frac{d_{1}}{2} B_{3}-\frac{5}{2} v_{2}^{2} B_{4}=0 \\
& \frac{d_{1}}{2} B_{2}+\left(b_{1}-9+\frac{7}{2} v_{2}^{2}\right) B_{3}+\frac{d_{1}}{2} B_{4}-\frac{9}{2} v_{2}^{2} B_{5}=0
\end{aligned}
$$

For $n \geq 2$

$$
\begin{aligned}
& -\frac{n(2 n-3)}{2} v_{2}^{2} B_{2 n-2}+\frac{d_{1}}{2} B_{2 n-1} \\
& +\left(b_{1}-4 n^{2}+\left(2 n^{2}-1\right) v_{2}^{2}\right) B_{2 n}+\frac{d_{1}}{2} B_{2 n+1} \\
& -\frac{n(2 n+3)}{2} v_{2}^{2} B_{2 n+2}=0 \\
& -\frac{n(2 n-1)-1}{2} v_{2}^{2} B_{2 n-1}+\frac{d_{1}}{2} B_{2 n}+ \\
& \left(b_{1}-(2 n+1)^{2}+\frac{\left.(2 n+1)^{2}-2\right)}{2} v_{2}^{2}\right) B_{2 n+1} \\
& +\frac{d_{1}}{2} B_{2 n+2}-\frac{n(2 n+5)+2}{2} v_{2}^{2} B_{2 n+3}=0
\end{aligned}
$$

\section{Even $4 \pi$-periodic solution}

Substitution a solution in the form

$$
\theta=\sum_{n=0}^{\infty} C_{n} \cos \left(n+\frac{1}{2}\right) \beta
$$

In equation (40) yields the recurrence relations for coefficients $C_{n}$

$$
\begin{array}{ll}
\left(b+\frac{d}{2}-\frac{1}{4}-\frac{7}{8} v_{1}^{2}\right) C_{0}+\left(\frac{5}{16} v_{1}^{2}+\frac{d}{2}\right) C_{1}-\frac{7}{16} v_{1}^{2} C_{2}=0 & -\frac{(2 n-5)(2 n+1)}{16} v_{1}^{2} D_{n-2}+\frac{d}{2} D_{n-1}+ \\
\left(\frac{d}{2}+\frac{9}{16} v_{1}^{2}\right) C_{0}+\left(b-\frac{9}{4}+\frac{1}{8} v_{1}^{2}\right) C_{1}+\frac{d}{2} C_{2}-\frac{27}{16} v_{1}^{2} C_{3}=0 & \left.+\frac{(2 n+1)^{2}}{4}+\frac{(2 n+1)^{2}-8}{8} v_{1}^{2}\right) D_{n} \\
& +\frac{d}{2} D_{n+1}-\frac{(2 n+1)(2 n+7)}{16} v_{1}^{2} D_{n+2}=0
\end{array}
$$

and substituting in (40) yields the following recurrence relations for coefficients $D_{n}$

$$
\begin{aligned}
& \left(b-\frac{d}{2}-\frac{1}{4}-\frac{7}{8} v_{1}^{2}\right) D_{0}+ \\
& \left(\frac{d}{2}-\frac{5}{16} v_{1}^{2}\right) D_{1}-\frac{7}{16} v_{1}^{2} D_{2}=0 \\
& \left(\frac{d}{2}-\frac{9}{16} v_{1}^{2}\right) D_{0}+\left(b-\frac{9}{4}+\frac{1}{8} v_{1}^{2}\right) D_{1} \\
& +\frac{d}{2} D_{2}-\frac{27}{16} v_{1}^{2} D_{3}=0
\end{aligned}
$$$$
\text { are }
$$$$
\left(b_{1}+\frac{d}{2}-\frac{1}{4}-\frac{7}{8} v_{2}^{2}\right) C_{0}+
$$$$
\left(\frac{5}{16} v_{2}^{2}+\frac{d_{1}}{2}\right) C_{1}-\frac{7}{16} v_{2}^{2} C_{2}=0
$$$$
\left(\frac{a_{1}}{2}+\frac{9}{16} v_{2}^{2}\right) C_{0}+\left(b_{1}-\frac{9}{4}+\frac{1}{8} v_{2}^{2}\right) C_{1}
$$$$
+\frac{d_{1}}{2} C_{2}-\frac{27}{16} v_{2}^{2} C_{3}=0
$$

For $n \geq 2$

$$
\begin{aligned}
& -\frac{(2 n-5)(2 n+1)}{16} v_{2}^{2} C_{n-2}+\frac{d_{1}}{2} C_{n-1}+ \\
& \left(b_{1}-\frac{(2 n+1)^{2}}{4}+\frac{(2 n+1)^{2}-8}{8} v_{2}^{2}\right) C_{n} \\
& +\frac{d_{1}}{2} C_{n+1}-\frac{(2 n+1)(2 n+7)}{16} v_{2}^{2} C_{n+2}=0
\end{aligned}
$$

Odd $4 \pi$-periodic solution

Assuming such solution in the form

$$
\theta=\sum_{n=0}^{\infty} D_{n} \sin \left(n+\frac{1}{2}\right) \beta
$$


While for the second case the recurrence relations are

$$
\begin{aligned}
& \left(b_{1}-\frac{d_{1}}{2}-\frac{1}{4}-\frac{7}{8} v_{2}^{2}\right) D_{0}+ \\
& \left(\frac{d_{1}}{2}-\frac{5}{16} v_{2}^{2}\right) D_{1}-\frac{7}{16} v_{2}^{2} D_{2}=0 \\
& \left(\frac{d_{1}}{2}-\frac{9}{16} v_{2}^{2}\right) D_{0}+\left(b_{1}-\frac{9}{4}+\frac{1}{8} v_{2}^{2}\right) D_{1}+ \\
& \frac{d_{1}}{2} H_{2}-\frac{27}{16} v_{2}^{2} D_{3}=0
\end{aligned}
$$

For $n \geq 2$ :

$$
\begin{aligned}
& -\frac{(2 n-5)(2 n+1)}{16} v_{2}^{2} D_{n-2}+\frac{d_{1}}{2} D_{n-1}+ \\
& \left(b_{1}-\frac{(2 n+1)^{2}}{4}+\frac{(2 n+1)^{2}-8}{8} v_{2}^{2}\right) D_{n} \\
& +\frac{d_{1}}{2} D_{n+1}-\frac{(2 n+1)(2 n+7)}{16} v_{2}^{2} D_{n+2}=0
\end{aligned}
$$

The systems of equations $(43,46,49,52)$ and also $(44,47,50,53)$ are homogeneous infinite systems. The boundary curves can be drawn by solving the determinantal equations. We shall study the stability and instability zones in the plane of parameters $\alpha_{0} \psi_{0}$ where $\psi_{0}$ is the amplitude of vibrations $\left(v_{1,2}=\sin \psi_{0}\right)$.

\section{Numerical and analytical diagrams of stability}

Let the symbols $W_{s}, G_{s}$ denote the (s) zones of stability and instability, respectively, separated by the curves $\prod_{2 s}^{ \pm}, \prod_{2 s+1}^{ \pm}$whose equations describe the distribution of eigen-values for boundary problems of periods $2 \pi, 4 \pi$. We shall draw these curves by solving the last four determinants. We classify the curves as:

$$
\begin{aligned}
& \prod_{2 s}^{+} \text {for even } 2 \pi \text { - periodic solutions and } \\
& \prod_{2 s}^{-} \text {for odd } 2 \pi-\text { periodic solutions. } \\
& \prod_{2 s+1}^{+} \text {for even } 4 \pi-\text { periodic solutions and } \\
& \prod_{2 s+1}^{-} \text {for odd } 4 \pi-\text { periodic solutions. }
\end{aligned}
$$

As a first step we now find the intersections of $\prod_{2 s}^{ \pm}, \prod_{2 s+1}^{ \pm}$with the line $\psi_{0}=0$. On this line the equation of vibration (36) takes the form

$g^{\varpi}+\left[1+\frac{1}{3(1-\alpha)}\right] g=0$

let $1+\frac{1}{3(1-\alpha)}=\omega^{2}$ where $\omega$ is constant, then $\alpha=1-\frac{1}{3\left(\omega^{2}-1\right)}$, for $\omega=2,3,4,5,6, \ldots$.

$\alpha_{1}=\frac{8}{9}, \alpha_{2}=\frac{23}{24}, \alpha_{3}=\frac{44}{45}, \alpha_{4}=\frac{71}{72}, \alpha_{5}=\frac{104}{105}, \ldots \ldots$

and so on. There exist two curves with the same period one even and other odd passing through each of those values of $\alpha$. We note that the stability zones occupy the lower part near to $\Psi_{0}=0$. For example, two curves, one odd $\prod_{2 s+1}^{-}$ and the other even $\prod_{2 s+1}^{+}$. with the same period $4 \pi$ at $\alpha=\frac{8}{9}$ and $\prod_{2 s}^{+}, \prod_{2 s}^{-}$with period $2 \pi$ at $\alpha=\frac{23}{24}$ (see Fig. 5).

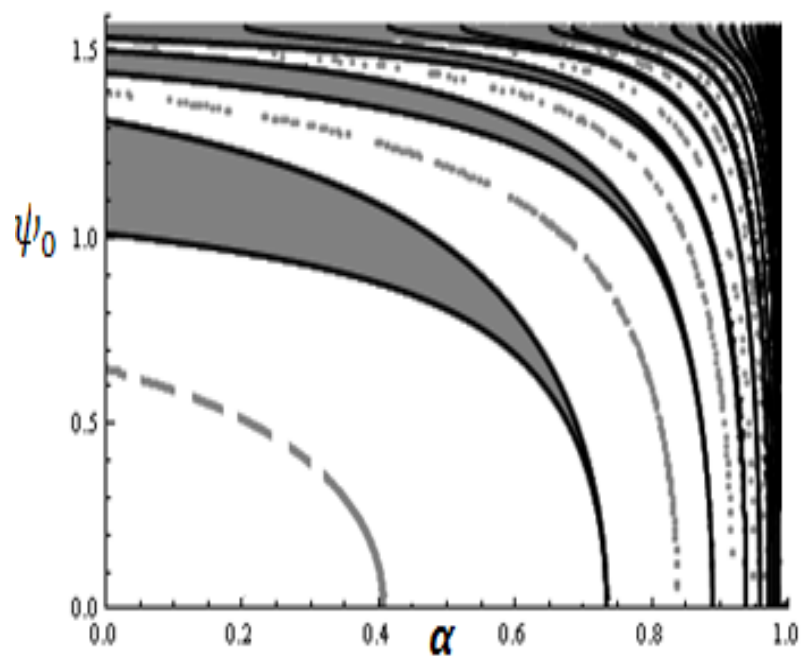

Fig. 5 stability zones for the case I

In the same manner as in the case of $(0<\alpha<1)$ we get the intersections of $\prod_{2 s}^{ \pm}$, $\prod_{2 s+1}^{ \pm}$with $\psi_{0}=0$ to determine the zones of stability and instability for the case $(1<\alpha<2)$

Equation (39) becomes: 
$g+\left[1+\frac{1}{3(\alpha-1)}\right] g=0$

Let $\frac{1}{3(\alpha-1)}=n^{2}$ where $n(+)$ ve integer, then $\alpha=\frac{1}{3 n^{2}}+1$ for $n=1,2,3,4, \ldots$.

$\alpha_{1}=\frac{4}{3}, \alpha_{2}=\frac{13}{12}, \alpha_{3}=\frac{28}{27}, \alpha_{4}=\frac{49}{48}, \ldots \ldots$

and so on. The zones of stability and instability are shown in Fig. 6.

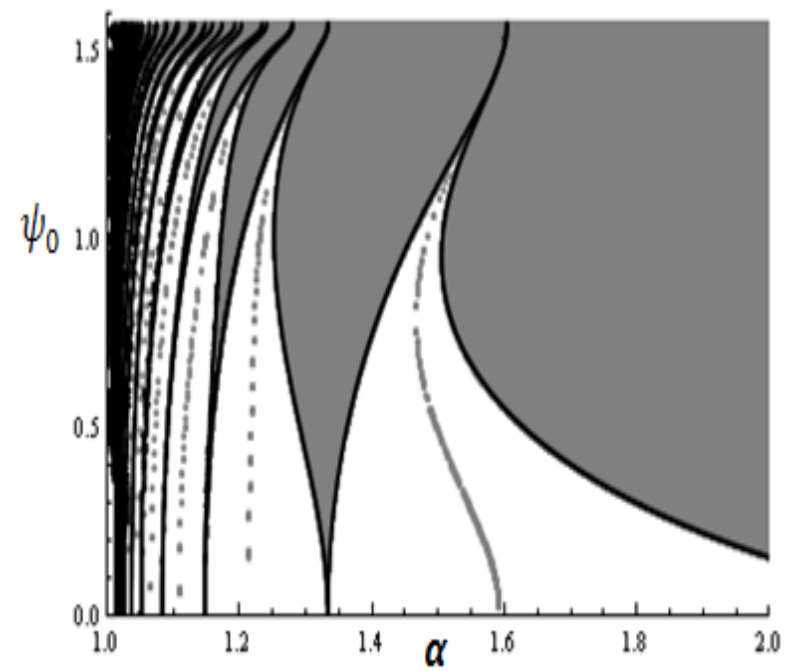

Fig. 6 stability zones for the case II

\section{Conclusion}

The stability of the vibrational in-plane motion of the satellite in a circular orbit is studied. The plane of parameter $\left(\alpha, \psi_{0}\right)$ is divided into distinct regions of stability and instability separated by certain curves corresponding to four types of periodic solutions of Hill's equations. Those zones are illustrated graphically.

\section{References}

[1] E. Leimanis, The General Problem of Motion of Coupled Rigid Bodies About a Fixed Point. Springer-Verlag (1965)

[2] J. Awrejcewicz, Classical Mechanics. Dynamics, Springer, Lodz (2012)

[3] V.V. Beletskii, Motion of an Artificial Satellite about Its Center Of Mass. Nauka. Moscow (In Russian) (1965)

[4] H.M. Yehia, On the motion of a rigid body acted upon by potential and gyroscopic forces, J. Mecan. Theor. Appl. 5 (1986) 747754

[5] A.P. Markeev, Stability of plane oscillations and rotations of a satellite in a circular orbit, Kosmich. Issled. 13 (1975) 322-336 (in Russian), English transl. Cosmic Res. 13 $285-298$

[6] L.D. Akulenko, S.V. Nesterov, A.M. Shmatkov, Generalized parametric oscillations of mechanical systems, Prikl. Mat. Mekh. 63, 746-756 (in Russian); English transl J. Appl. Math. Mekh. 63 (1999) 705-713

[7] A.P. Markeev, Bardin, On the stability of planar oscillations and rotations of a satellite in a circular orbit, Celest. Mech. Dyn. Ast. 85 (2003) 51-66

[8] F.M. Arscott, Periodic Differential Equations. Pergamon Press, Oxford (1964) 